\title{
Enhancing Inertia of a Grid Connected PV System to Improve Frequency Regulation and Stability
}

\author{
Jayaprakash Allemshetty \\ Department of Electrical and \\ Electronics Engineering \\ University College of Engineering, \\ Osmania University \\ Hyderabad, India. \\ jayaprakashallemshetty@gmail.com
}

\author{
B. Mangu \\ Department of Electrical and \\ Electronics Engineering \\ University College of Engineering, \\ Osmania University \\ Hyderabad, India. \\ bmanguou@gmail.com
}

\author{
Kalluri Manaswee Reddy \\ Department of Electrical and \\ Electronics Engineering \\ University College of Engineering, \\ Osmania University \\ Hyderabad, India. \\ k.manaswee.reddy@gmail.com
}

\begin{abstract}
Power generation from solar photovoltaics is rapidly increasing. In this scenario, this new technology has to work with the existing system in tandem to maintain stability during contingencies as it is a low inertia system. The interfacing power electronics devices have to be designed in such a way that the integration of the photovoltaic (PV) to the Grid is to be taken care during the disturbances. This paper presents the various issues when the PV penetration is comparable with the system capacity. The frequency of the system drops more in the PV-Grid connected system than the conventional generation system for same disturbance. Also, the frequency regulation methods get affected. The mode of operation of the inverter might be altered. An auxiliary source like batteries, ultra-capacitors is being suggested to balance the power for successful operation during abnormalities. In spite of this, many authors had developed new control techniques to mimic the interfacing inverter like conventional generating unit. The basic concepts of these methods have not explained in most of the literature. This paper reviews the concepts and applications of these methods and compares them.
\end{abstract}

Keywords - Inertia, droop characteristics, Stability, Grid, Control Parameters, Interfacing, Grid Integration.

\section{INTRODUCTION}

In the recent years, there is a huge deputation of solar PV cells throughout the world due to various reasons like renewable, eco-friendly, localized generation, and easy installation and even due to its modularity. In India the energy generated through PV cells is around 132.41 MU. [1] Even the Govt. of India has started International Solar Alliance to promote the generation electrical energy utilizing solar energy. It has targeted to generate $100 \mathrm{GW}$ by the year 2022 by only solar PV power under National Solar mission. This comprises of around 22-25\% of total Energy generated in India [1]. As per the reports some of the thermal units are going to be shut down in future [2]. Therefore, this transition will make certain impact on the present existing power system operation and control. Either the existing power system is renovated or a new power system is to be developed in mere future. New technologies in all the components of power system have to be adopted to meet the future requirements. The concept of Microgrids, Smart Micro grids and DC Microgrids [3] are some of the emerging changes in the power system. As the penetration level of PV power is increasing into the grid, apart from adopting new technologies, the stability of the grid is also a major concern. Literature studies identified the stability of the power system may be affected with the penetration of large PV power. The basics of the power system stability reveal that the inertia of the generators is an important parameter in maintaining the stability of the power system under contingencies. The inertia of the existing generation units is good enough to maintain the stability but with the intervention of the less inertia generators like PV the stability of power system get affected [4]. The other major drawback of PV energy is reliability. The energy from it depends on the weather conditions. The irradiance and insolation of the sun is not constant throughout the day [5]. Hence this behaviour also causes stability problems as PV power penetration is high.

The researchers have been proposing methods for improving stability of the PV, grid connected system for the past few years. These methods can be widely classified into two major categories. First category is to adopt an energy storage device or a generator which delivers or absorbs the mismatch energy during the disturbances like battery storage system, diesel generators etc. [8]- [15]. The presence of other generators mainly those with rotating parts will improve the net inertia of the system apart from maintaining the power balance during the disturbances. Second category is to modify the operation of the interfacing power electronic converters to emulate as a synchronous generator. This method is popularly known Virtual Inertia, synchro-converter [16]- [26] etc. This paper mainly concentrates few of the methods proposed by the researches to improve the transient stability of the PV - Grid connected system. The paper is organized as follows. Section II describes the concept of virtual inertia from the principles of power system stability. Section III deals with few methods by using energy storage system (ESS) or an auxiliary source. Section IV describes the second category methods to improve the inertia and the converter topologies as other choice.

\section{VIRTUAL INERTIA}

\section{A. Swing Equation}

A conventional synchronous generator is driven by turbine with a constant speed. The Kinetic Energy (K.E) of the rotor of synchronous generator [6] is given as (1). Where $\mathbf{J}$ is moment of inertia of rotor in $\mathrm{kg} / \mathrm{m}^{2}, \omega_{\mathrm{s}}$ is the synchronous speed in $\mathrm{rad} / \mathrm{sec}$.

$$
K . E=\frac{1}{2} J \omega_{s}^{2} \times 10^{-6}
$$

The torque equation governing the dynamics of rotor is given by equation (2)

$$
J \frac{\partial^{2} \theta_{m}}{\partial t^{2}}=T_{m}-T_{e}
$$




$$
M \frac{\partial^{2} \theta_{m}}{\partial t^{2}}=P_{m}-P_{e}
$$

Where, $\theta_{m}$ is the displacement angle between rotor torque, $\mathrm{T}_{\mathrm{m}}$ and electromagnetic torque $T_{e}, M$ is the moment of inertia in MJ-s/elect.rad. Consider the inertia constant of a synchronous generator defined as the amount of K.E stored corresponding to its rating in MVA $(\mathrm{S})$. For a given rating if the value of ' $\mathrm{H}$ ' is high, the kinetic energy stored in the machine is also more at a particular angular speed.

$$
H=\frac{K . E}{S} M J / M V A
$$

The equation (5) is obtained by substituting ' $M$ ' in terms of inertia constant ' $\mathrm{H}$ '.

$$
\frac{H}{\pi f} \frac{\partial^{2} \theta_{m}}{\partial t^{2}}=P_{m}-P_{e}=\Delta P
$$

Where, ' $\delta$ ' is called rotor displacement angle from synchronously rotating frame and popularly known as torque angle or power angle. Equation (5) is called as the swing equation and it illustrates the dynamics of the rotor. From equation (1) - (5), we can conclude that, when there is a change in load, ( $\triangle P$ may be positive or negative) the rotational speed of the prime mover changes and thus K.E of the turbine accordingly. This change in K.E with respect to the time results is the power generated or absorbed which is necessary to keep system power in balance at the cost of change in the frequency. Hence the system power is balanced until the valve, automatic governor control (AGC) comes into action. This automatic power balance is not much possible with the less inertia, static power electronic interfaced generators.

In a multi machine system, where there are many generators the total inertia of the system is shown [7]

$$
\frac{\prod^{n} H_{n}}{\sum_{i=1}^{n} H_{n}} M J / M V A
$$

Where $H_{n}$ is the inertia constant of individual unit. The rate of fall of frequency when there is disturbance or mismatch in the power is given in the equation (7) which is derived in the reference [7]

$$
\frac{d f}{d t}=\frac{f_{0}}{2 H_{e q}} \frac{\Delta P_{L}}{\sum P_{n i}}
$$

In the paper [8], the author presented the inertia analysis on the conventional speed governor model which is similar to the figure 1 . He claimed that ' $H$ ' has no much impact in quasi-steady state frequency change but it certainly has impact on Rate of Change of Frequency (RoCoF) and frequency nadir. As the PV power penetration increases into the grid, the net inertia of the system get decreased and from equation (6) and (7), we can conclude that with the increase in PV penetration, the less inertia generator, the rate of fall of frequency will increase for the same amount of power

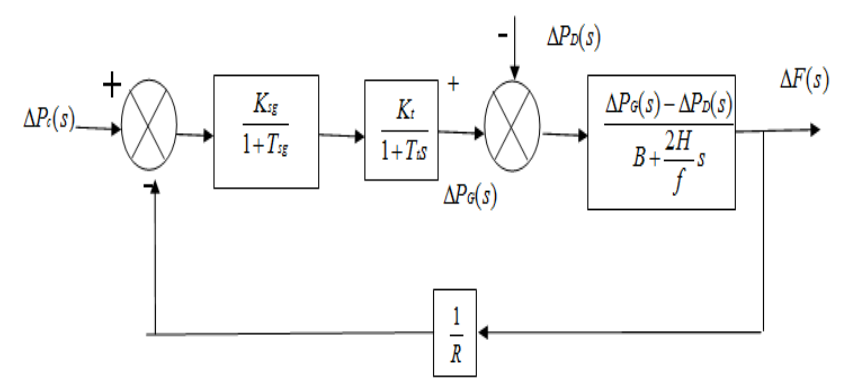

Fig. 1: Turbine-Governor model of a conventional system

mismatch when compared to a conventional system than PVGrid integration system.

\section{B. Turbine-Governor Model}

In Figure 1, the turbine governor model of a synchronous generation is described. In the figure, $\Delta P_{c}(s)$ is the power command given by the turbine, $\Delta P_{G}(s)$ is the power delivered by the generator and $\Delta P_{D}(s)$ is the load demand.

$R$ represents the speed regulation of the governor which is approximated as

$$
\Delta f=-R \Delta P_{D}
$$

The fall of frequency when there is a change in power balance is controlled by the speed governor $R$. The P-f droop control governor will increase the mechanical input power and settles at steady frequency which may be lower than nominal value. This mechanism doesn't exist in PV system as there are no rotating parts. The rate of change of frequency (RoCoF) with respect to rate of change of power mismatch is obtained from figure1. If the PV system is made to mimic as a speed turbine governor model as described above by introducing inertia constant called Virtual Inertia and controlling power by droop control method called as Virtual Synchronous Generator, the PV-Grid system may resemble as conventional power system and the stability of the system may remain same. The concept of virtual inertia is introduced in balancing the power following droop characteristics. The inverter and other converters are operated by the references from the droop characteristics. The researchers are proposing many methods in this methodology to attain stability in the PV-Grid connected system.

\section{STABILITY IMPROVEMENT USING ESS OR ALTERNATE ENERGY SOURCE}

In this method an energy storage system is utilised or an alternate energy source like a diesel generator, wind turbine is placed. In energy storage system like battery and capacitor the power is absorbed or delivered. The following principle is presented in the paper [8] representing the concept of virtual inertia. The reference power from the converter of ESS $P_{E S S}^{*}$ is measured as given by the equation (8) [9]

$$
P_{E S S}^{*}=-K_{v i} K_{v r} f_{o} \Delta f
$$

Both the equations (7) and (9) are similar which is principle of conventional turbine governor model. $K_{v i}$ is the virtual inertia of the system, $K_{v r}$ is th conversion factor, $f_{0}$ is the nominal frequency. The design of $K_{v i}$ is very important to 


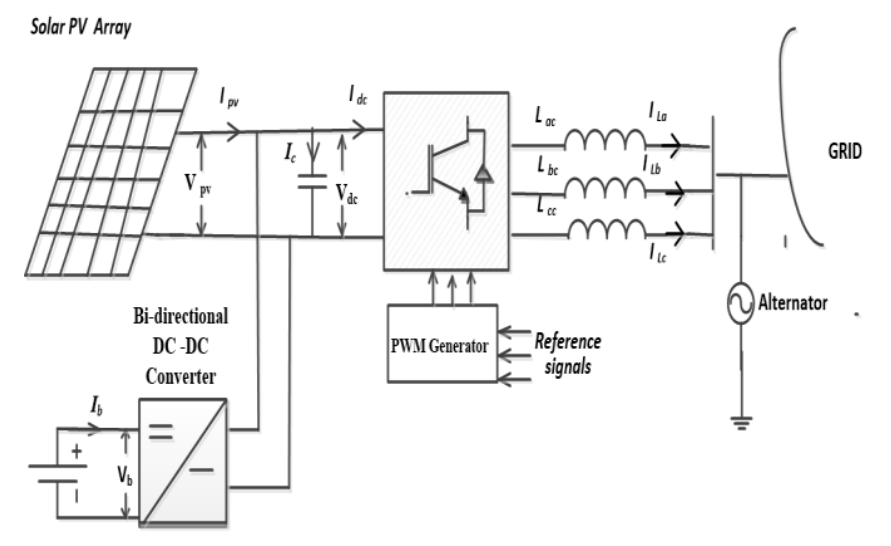

Fig. 2: Generalized PV system with Energy storage device. introduced. In this paper the synchronous machine is integrated with a hydro generator. The VSM may be a battery or super capacitor or PV. The integration of VSM with a hydro generator has less inertia. Hence the VSM is modelled to emulate as convention generator by designing the fall of active power with respect to frequency i.e., RoCoF as following equation.

$$
P_{V S M}=K_{I} \frac{d(\Delta f)}{d t}+K_{P}(\Delta f)
$$

$K_{I}$ and $K_{P}$ are inertia constant and damping constant respectively. First term in the equation (9) comes into picture during transients to reduce RoCoF whereas second term sets up the frequency to a reference value.

This concept of VSM is also implemented by considering by change in voltage popularly known as Rate of change of voltage $(\mathrm{RoCoV})$. The variation of power can also be estimated by looking at the variation in voltage [11] called as Rate of Change of Voltage (RoCoV). The power is estimated as.

$$
P_{V S M}=k_{i} \frac{s}{s+a} V_{d c}
$$

Where $\mathrm{P}_{\mathrm{vsm}}$ is the output power from the Virtual synchronous machine i.e., battery, $K_{i}$ (negative) virtual inertia which decides the amount of active power to be deliver and $\mathrm{Vdc}$ is washout filter output voltage whose input is dc link voltage at the inverter. The parameter are chosen from the stability analysis will vary from researcher to other researcher.

Apart from considering the power, frequency and voltage, the impedance is also considered in the paper [12] called as virtual impedance in the droop control strategy. The virtual reactance is placed in the following equation given as

$$
\begin{aligned}
& V_{d}=-R_{v} i_{d}-L_{v} \frac{d i_{d}}{d t}+\omega L_{v} i_{q}+V \\
& V_{q}=-R_{v} i_{q}-L_{v} \frac{d i_{q}}{d t}+\omega L_{v} i_{d}+0
\end{aligned}
$$

Where $R_{v}$ and $L_{v}$ are virtual resistance and virtual reactance respectively. The equations mentioned above are used to control the inverter. In this way the concept of virtual inertia was being implemented.
In the paper [13], The concept of synthetic inertia is proposed along with the Modular Multilevel Converters (MMC). In this work, the sub-modules capacitors energy is used which in turn used as synthetic inertia. An MMC with inertia constant is presented. The author controlled the frequency droop when there is change in the load by utilizing the synthetic inertia. The fall in frequency in conventional synchronous generator as discussed earlier is compared with the reduction in the voltage of sub-modules capacitors. A reference voltage $\Delta U_{c}{ }^{*}$ of the sub-module capacitors is generated by considering the fall in the frequency by the equation (13) which is similar to (3). $U_{c}$ and

$\Delta \omega$ are the DC voltage and change in frequency in $\mathrm{rad} / \mathrm{sec}$ respectively.

$$
\Delta U_{c}^{*}=\frac{\beta}{\lambda} U_{c} \Delta \omega
$$

The change in the energy with the change in the increase or decrease in sub-modules voltage is shown in the equation (13). This energy change is used to generate the reference for active power variation since the load change will be in-terms of active power. The experimental results proved the energy in the capacitors can be used for short duration of time to cope the power variations.

$$
\Delta E_{s m}=\frac{1}{2} C_{s m}\left(U_{c}+\Delta U_{c}\right)^{2}-\frac{1}{2} C_{s m} U_{c}^{2} \approx \frac{C_{s m} U_{d c}^{2}}{N^{2}} \frac{\beta}{\lambda} \Delta \omega
$$

The author in this paper [14] estimated the capability of the static interfacing power electronic converters in maintain the frequency when there is change in the load of $3 \%$. The work is done on a microgrid i.e., ship board power structure of 1 MVA. In the paper the author made a close summary on the limitation on the utilization of dc link capacitor as an energy storage which delivers the power. The results show the RoCoF is within the limits but there is reduction of the DC link voltage. For a microgrid, the DC link voltage can be increased by other sources.

\section{STABILITY IMPROVEMENT BY INVERTER ALONE}

The concept of synchronous generator, virtual inertia can

be implemented without any energy storage or auxiliary localized synchronous generator. The Inverter itself is designed to mimic as a conventional turbine -governor synchronous generator model. The inverter will act as a source of active and reactive power. The output of the inverter is given as shown [16] for a microgrid where $\mathrm{R}>>$ $\mathrm{X}$. From above two equations the active power and reactive power can be controlled by Ui and $\delta$ i by choosing a reference and PLL. Both the powers can be controlled independently. This method doesn't mimic as synchronous generator.

$$
\begin{aligned}
P_{i} & =\frac{V}{\left|Z_{i}\right|}\left[U_{i}-V\right] \\
Q_{i} & =\frac{U_{i} V}{\left|Z_{i}\right|} \delta_{i}
\end{aligned}
$$

The droop coefficient has to be included to control the power to make inverter to function as virtual synchronous generator. 
In the paper [17], the control mechanism is explained by introducing droop coefficient.

$$
\begin{gathered}
U_{i}=U_{i}^{*}-K_{p i}\left(P_{i}-P_{i}^{*}\right) \\
f_{i}=f_{i}^{*}+K_{q i}\left(Q_{i}-Q_{i}^{*}\right)
\end{gathered}
$$

Where $\mathrm{K}_{\mathrm{pi}}$ and $\mathrm{K}_{\mathrm{qi}}$ are the droop coefficients $f_{i}^{*}, U_{i}{ }^{*}$ are the reference values and $P_{i}^{*}$ and $Q_{i}^{*}$ are the reference set values. Equation (13) is used to control the active power whereas equation (14) is used to control the reactive power in the system. The reason to choose the frequency as a control variable for reactive power control is that the droop coefficients are not easily determined and even phase angle also. Phase angle can be measured from frequency as we do in PLL. It is expressed as integral of

frequency which can realized from the below equation (15)

$$
\begin{aligned}
u_{i}(t) & =\sqrt{2} U_{i} \sin \delta_{i} \\
& =\sqrt{2} U_{i} \sin \left(\int_{0}^{t} 2 \pi f(\tau)+\delta_{i o}\right)
\end{aligned}
$$

Where, dio is the initial phase angle set for inverter. The control of reactive power using frequency droop is explained in the paper. The dynamic performance and stability of a VSG not only depends on inertia but also damping ratio. In the paper [18], the author explains the necessity of control of damping ratio along with droop gain method. The author in [18] proposed a control scheme which provides freedom in tuning the damping ratio from droop gain. This will reduce the steady state error caused by the droop gain since a decoupled method is followed in the paper. This method is used to control both active and reactive power in the system. A small signal analysis was proposed for the controller to study the system stability against system frequency and voltage variations which gives guidelines for the design of control parameters. The impact of high penetration of PV power was described in paper [19]. In this work a $100 \mathrm{MW}$ PV plant was considered which is connected a northern Chile where the average demand is around $2000 \mathrm{MW}$ and its stability was observed. Each inverter was designed to act as synchronous generators by imparting synchronous power controller methodology. The author observed the performance for small and large disturbances in the power system. There are two controllers, one the central plant controller and the other is converter controller. The central plant controller will generate set points of active and reactive power taking an account of frequency at the point of common coupling (PCC). The voltage is controlled by generating a reactive power reference at the PCC. The converter controller emulates the electromagnetic force of the virtual synchronous generator behind a virtual admittance. The author claimed requirement of PLL is not necessary using this method. A current reference was generated which will flow the virtual admittance which results in voltage difference between the internal virtual EMF and the voltage at PCC where the converter was connected. The converter controller includes frequency and voltage droops that modifies the active and reactive power references from the central controller. The dynamics of PV power variation was not included in this paper such as variation of irradiation, temperature and dc

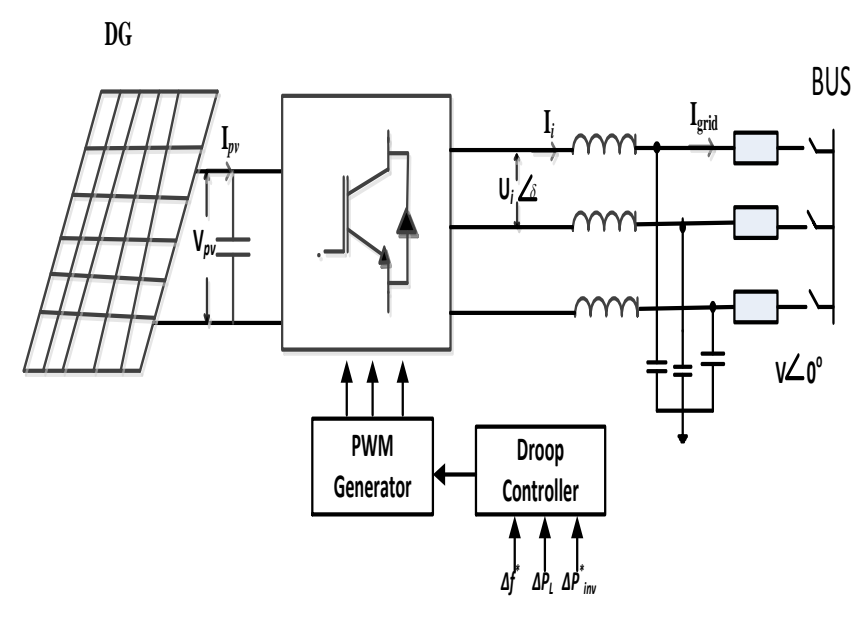

Fig 3: A Generalized PV-Grid system without energy storage

voltage, since these variations are much slower than the grid variations. Hence maximum power point tracking (MPPT) methods are not employed in this paper. In the work the PV were designed to contribute to frequency regulation with a $5 \%$ droop and the synchronous power controller (SPC) an inertia constant of 5s. The study evaluated for different cases like line contingency, generator contingency, load contingency. Finally, the author claimed that there were less severe oscillations when SPC was enabled in the real time application of 100 MW PV plant on 2000 MW power system.

To emphasis the method of virtual inertia among the converter, the author [20] proposed a derivative control based virtual inertia in multi-area HVDC interconnected system. The method was used to suppress the first overshoot which was tested in simulation. In fact, in HVDC system converter are used which has no inertia. In this paper, the derivative of the system frequency was obtained and the power reference for converter was modified. In his study, and energy storage system is used for emulating inertia. The analysis was made for different control parameters.

In paper [21], the author proposed a control law which is derived from the structure preserving energy function (SPEF). The energy preserving function is applied for SSSC, SVC, and STATCOM as given in the literature. In the work author claimed that by controlling the reactive current component of PV inverter can damp the oscillations and maintain the stability. The reactive current component is a dependent on the PV voltage, which is measured continuously. Therefore, in the work, the reactive component or reactive power is controlled by keeping in the view of stability.

\section{CONCLUSION}

An attempt was made to analyze various research papers and review them in detail. A clear understanding of the significance of inertia is also realized in this paper. The very primitive idea of this paper is to overcome the difficulty of understanding the stability issues system in PV-Grid integrated system. A comparative study is given out, in 
improving inertia of the system by the researchers in which various techniques proposed by them to enhance the inertia are mentioned. This paves the way for simplicity in designing the control methods by inserting inertial parameters to mimic as conventional generator. The paper classifies two major methods and describes some of the solutions. Though many researchers have provided the methodologies, at the higher levels of PV power injection the efficient methods are to be developed and to be proved experimentally. As this scope of renewable sources widely renowned, innovating the interfacing power electronic devices that are currently in use by adopting the methodologies in literature would certainly pose a challenge that is yet to be solved. The utilities able to adopt these methods at a faster rate for the successful integration.

\section{REFERENCES}

[1] Website of Ministry of New and Renewable Energy Sources,2019 [online], http://www.mnre.gov.in/scheme-document [accessed on 1111-2019]

[2] National Electricity Plan, 2018.

[3] Nikos Hatziargyriou, "Microgrids: Architecture and Control", First Edition, John Wiley \& Sons, Ltd. 2014

[4] R. Yan, T. K. Saha, N. Modi, N. Mosadegh, “ The Combined Effects of High Penetration of Wind and PV on Power System Frequency Response", Applied Energy, vol.145,pp.320-330, May 2015

[5] Dr. Ajit P. Tyagi, "Solar Radiant Energy over India”, 2009

[6] D P Kothari, I J Nagrath “ Modern Power System Analysis” third edition Tata McGraw Hill Education Pvt. Ltd.

[7] Pablo F. Frack, Pedro E. Mercado, Marcelo G. Molina, Edson H. Watanabe, Rik W. Doncker and Hanno Stagge "Control Strategy for Frequency Control in Autonomous Microgrids", IEEE Journal of Emerging and Selected topics in Power Electronincs, Vol. 3, No. 4,

[8] Jingyang Fung, Hongchang Li, Yi Tang, Frede Blaabjerg, "Distributed Power System Virtual Inertia Implemented by GridConnected Power Converters", IEEE Transactions on Power Electronics, Vol. 33, No. 10 , October 2018, pp 8488 - 8499

[9] Manoj Datta, “ Frequency Control Improvement in a PV- Diesel Hybrid Power Ssytem with a Virtual Inertia Controller"

[10] Ujjwol Tamrakar, David Galipeau, reinaldo Tonkoski, Indraman Tamrakar, " Improving Transient Stability of Photovoltaic-Hydro Microgrids Using Virtual Synchronous Machines,

[11] Ali Hosseinpour, Hossein Hojabri, "Virtual inertia control of PV systems for dynamic performance and damping enhancement of DC microgrids with constant power loads", IET Renewable Power Gener., 2018, Vol. 12, Iss. 4, pp. 430-438.
[12] Zishun Peng, Jun Wang, Daqiang Bi, Yeting Wen, Yuxing Dai, Xin Yin, Z John Shen, “ Droop Control Strategy Incorporating Coupling Compensation and Virtual Impedance for Microgrid Application"

[13] Shunfeng Yang, Jingyang Fang, Yi Tang, Huan Qiu, Chaoyu Dong, Peng Wang, " Modular Multilevel Converter Synthetic Inertia-based Frequency Support for Medium -Voltage Microgrids” IEEE Trans. on Industrial Electronics. DOI 10.1109/TIE.2018.2890491

[14] Huan Qiu, Jingyang Fang, Yi Tang "Explore the capability of Power Electronic Converters in Providing Power System Virtual Inertia"

[15] Francisco Sanchez, Joshua Cayenne, Francisco Gonzalez-Longatt, Jose Luis Rueda, "Controller to enable the enhanced frequency response services from a multi-electrical energy storage system, IET Gener. Transm. Distrib., 2019 Vol. 13 Iss. 2, pp. 258-265.

[16] Gaber Magdy, G. Shabib, Adel A. Elbase, Yasunori Mitani, “A Novel Coordination Scheme of Virtual Inertia Control and Digital Protection for Microgri Dynamic Security Considering High Renewable Energy Penetration", IET Renew. Power Gener., 2019, Vol 13 Iss 3, pp. 462 474.

[17] Chunfeng Gao, Rengang Yang, Jiao Jiao, zhenhai Dou “ Power Control Strategy Design in an Islanded Microgrid based on Virtual Frequency",

[18] Elyas Rakhshani, Daniel Remon, Antonni Mir Cantarellas, Pedro Rodriguez, " Analysis of derivative control based virtual inertia in multo-area high-voltage direct current interconnected power systems", IET Gener., Transm. \& Distrib., 2015

[19] Daniel Remon, Claudio Canizares, Pedro Rodriguez, "Impact of 100MW-scale PV plants with synchronous power controllers on power system stability in northern Chile, IET Gener., Transm. \& Distrib., 2017, Vol. 11, Iss 11, pp. 2958-2964.

[20] Arash Asrari, Mehran Mustafa, Meisam Ansari and Javad Khazaei "Impedance Analysis of Virtual Synchronous Generator- Based Vector Controlled Converter for Waek AC Grid Integration"

[21] Rupesh G. Wandhare, Vivek Agarwal, “ Novel Stability Enhancing Control Strategy for Centralized PV-Grid Systems for Smart Grid Application"

[22] Yawei Wang, Bangyin, Shanxu Duan "Modified Virtual Inertia control Method of VSG Strategy with Improved transient response and power-supporting capability" IET Power Electron. ISSN 17554535.

[23] Junru Chen, Terence o'Donnell, “Analysis of virtual synchronous generator control and its response based on transfer functions" "IET Power Electron. ISSN 1755-4535.

[24] Jia Liu, Yushi Miura and Toshifumi ISE, "Fixed -Paramaeters Damping Methods of Virtual Synchronous Generator Control Using State Feedback” IEEE Access, vol.. 7, 2019, pp: 99177 -99190

[25] Zhijun Li, Xueyan Jia, “An Improved VSG Control Strategy Based on the Amplitude-frequency Cahracteriesitcs of Virtual Power", IEEE Access Vol 7, 2019 pp:101096-101105

[26] Tarek Younis, Mohamed Ismeil, Essam K. Hussain, Mohamed Orabi, "Improved single-phase self synchronised synchroconverter with enhanced dynamaics and current limitation capability", " IET Power Electron. ISSN 1755-4535, pp: 337-344 